\title{
Opiniones del gato Murr, de E.Th.A. Hoffmann, y la tradición del Bildungsroman
}

Fecha de recepción: 19/5/2020. Fecha de aceptación: 20/10/2020.

\section{Resumen}

A partir de la publicación de la novela de Goethe Los años de aprendizaje de Wilhelm Meister (1795/96), se consolida el género novelístico del Bildungsroman, que ocupará un lugar dominante en el sistema literario del Kunstperiode (período artístico). La importancia de esta novela para los escritores románticos se evidencia en la discusión crítica que suscitó y, especialmente, en la cantidad de novelas que escribieron como respuesta al modelo goetheano. Opiniones del gato Murr (1819/1821), de E. Th. A. Hoffmann (1776-1822), al cuestionar las bases del esquema narrativo del Bildungsroman, se opone tanto al prototipo del género como a las réplicas románticas, por lo que puede considerarse que marca el final de una etapa en la evolución de la novela alemana.

Palabras clave: formación; Romanticismo; novela.

E.Th.A. Hoffmann's Kater Murr and the tradition of the Bildungsroman

\begin{abstract}
The publication of Goethe's Wilhelm Meister's Apprenticeship (1795/96) consolidated the Bildungsroman as a novelistic genre, which would come to occupy a dominant position in the literary system of the Kunstperiode. The importance of this novel for Romantic writers is evidenced by the critical discussion it provoked and, especially, by the number of novels they wrote in response to the Goethean model. E. Th. A. Hoffmann's (1776-1822) Kater Murr (1819/1821) questions the basis
\end{abstract}


of the Bildungsroman's narrative scheme, opposing both the prototype of the genre and the romantic reformulations. Therefore, it can be seen as signalling the end of a stage in the evolution of the German novel.

Keywords: formation; Romanticism; novel.

En diciembre de 1819 se publica en Berlín el primer volumen de Opiniones del gato Murr (Lebens-Ansichten des Katers Murr nebst fragmentarischer Biographie des Kapellmeisters Johannes Kreisler in zufälligen Makulaturblättern), ${ }^{1}$ de Ernst Theodor Amadeus Hoffmann. El mismo mes, en la Universidad Imperial de Dorpat, el profesor de estética Karl Morgenstern da la primera definición sistemática del género Bildungsroman (novela de formación). La aparición simultánea de la parodia y de la teorización del género no debe sorprendernos como una irónica casualidad temporal, por el contrario, son dos manifestaciones de que el género se ha consolidado, de que ya es reconocible una estructura narrativa particular. La novela de Hoffmann cuestiona esta estructura narrativa. Tradicionalmente, los estudios sobre la relación de esta obra con el género del Bildungsroman se concentran en una de las dos secciones de la novela, en la autobiografía de Murr. ${ }^{2}$ Esta sección aparece evidentemente como una ridiculización del género desde que coloca como figura protagónica a un gato con un impulso formativo (Bildungstrieb). ${ }^{3}$ Pero, más allá de esto, nuestro objetivo será ver cómo esta sección identifica y recrea, de manera paródica la estructura narrativa básica del género. A su vez, consideraremos en nuestro análisis de la novela y su relación con el Bildungsroman la sección de Kreisler. Pretendemos demostrar que el vínculo con la novela de formación es un tema central en las dos secciones, y que los rasgos del género parodiados en la sección de Murr también aparecen cuestionados, con procedimientos diferentes, en la de Kreisler. Además, sostendremos que esta obra de Hoffmann no se opone al modelo fundador del género, Los años de aprendizaje de Wilhelm Meister (1795/96), de Goethe, en el mismo sentido en que lo hacen las novelas de los románticos alemanes. A partir de esto, y de la reconstrucción histórica del lugar del Bildungsroman en el sistema literario de la época, postularemos que Opiniones del gato Murr marca el final de una etapa en la evolución de la novela alemana.

En la primera parte de este trabajo, reseñaremos los estudios previos sobre la relación entre Opiniones del gato Murr y el Bildungsroman, los aportes fundamentales y los errores comunes en los que incurre esta bibliografía crítica, a nuestro criterio. Luego, nos ocuparemos de la sección de Murr, para definir cómo funciona

1 Para las citas de esta obra colocaremos solamente el número de página entre paréntesis, que corresponde a la edición indicada en la bibliografía.

2 Con las dos secciones, no nos referimos a los dos volúmenes (1819/1821), sino a la particularidad formal de la obra, que alterna dos relatos con dos narradores y dos protagonistas diferentes. La novela presenta de manera intercalada la autobiografía del gato Murr, completa y ordenada cronológicamente, y la biografía fragmentaria y desordenada del músico Johannes Kreisler. Como se explica en el prefacio del editor ficticio, el gato destruyó la biografía y usó las hojas "en parte como base de apoyo, en parte como secante. Estas hojas quedaron en el manuscrito, y con él por error fueron editadas, como pertenecientes al mismo" (126).

3 Abraham caracteriza a Murr con esta palabra (cfr. 154). 
la parodia del género, un punto muy señalado por la crítica, pero poco explicado formalmente. Aquí, también precisaremos qué lugar ocupaba el Bildungsroman en las discusiones contemporáneas sobre la novela, lo que nos permitirá comprender la posición de Opiniones del gato Murr en la serie literaria. Por último, desarrollaremos las múltiples relaciones, apenas exploradas, entre la sección de Kreisler y el Bildungsroman. En este punto, demostraremos el carácter unitario de la obra en su oposición al género, al reconocer que los mismos rasgos parodiados por Murr se retoman en esta sección. A su vez, aquí discutiremos uno de los lugares comunes más difundidos en los estudios de esta novela, que consiste en considerar a Kreisler como un típico héroe romántico, lo que promueve la inclusión de esta novela en el conjunto de las obras románticas anti-Meister.

\section{I}

El primer análisis específico sobre la relación de Opiniones del gato Murr y el género del Bildungsroman es el de Jürgen Jacobs, en su libro clásico sobre este género, Wilhelm Meister und seine Brüder. Untersuchungen zum deutschen Bildungsroman (1981 [1972]). Allí, postula que la novela de Hoffmann marca el final del género entendido en su acepción clásica (goetheana y hegeliana), y distingue dos razones principales para esto, que se desprenden de las dos partes de la novela (ibíd.: 150). La primera razón es que la parte de Murr es una parodia del modelo goetheano: "La idea de la formación se pone necesariamente en duda cuando se ejemplifica en el desarrollo de un gato doméstico" (ibíd.: 147). ${ }^{5}$ En segundo lugar, la parte de Kreisler se conecta de manera indirecta con el esquema del género, y se opone a este por el rechazo a adoptar un desenlace conciliador (para Jacobs, un requisito del género) para la historia del protagonista (ibíd.: 149ss.). Estas hipótesis fundamentales han sido casi unánimemente aceptadas por los críticos posteriores (Mahoney, 1988; Steinecke, 1992; Selbmann, 1994; Nehring, 1997; Kremer, 2009; Stephan, 2019), que continuaron las líneas de análisis embrionariamente planteadas aquí. ${ }^{6}$

Por un lado, Selbmann realiza un aporte importante al extenderse sobre el lugar particular de la novela de Hoffmann en la evolución literaria alemana. En primer lugar, al igual que Steinecke (1992: 950), enfatiza que Opiniones del gato Murr no

4 La oposición de esta novela a la idea clásica de Bildung ya había sido señalada por Singer (1963: 305) y Segebrecht (1967: 79) en trabajos valiosos que no recuperamos porque, a diferencia de Jacobs y sus sucesores, no se ocupan de ese tema de manera específica.

5 Las traducciones son nuestras cuando no se indica lo contrario en la bibliografía.

6 Una excepción es el artículo de James McGlathery "E. T. A. Hoffmann and the Bildungsroman", publicado en la -por lo demás- muy buena compilación de ensayos sobre el Bildungsroman editada por James Hardin, titulada Reflection and Action. La conclusión de McGlathery sobre Opiniones del gato Murr demuestra que tiene una noción muy vaga del género y, lo que es más grave, de la distancia entre la ficción literaria y la vida real del autor: "Hoffmann's tomcat novel also fails the test as a Bildungsroman - and even as a parody of that genre for an important formal reason as well. Like Sterne's Tristram Shandy, Tomcat Murr is a fictional autobiography, whereas a Bildungsroman demands third-person narration [...] At the same time, the Murr and Kreisler stories, taken together, are indeed Hoffmann's autobiography and confession, an ironic depiction of himself as composer and as author, as his music pupil Julia Marc's passionate lover from afar and as a subject to tomcat instincts as well" (1991: 327). 
se opone simplemente al género Bildungsroman, sino a la idea misma de Bildung, lo que significa un verdadero cambio de época (Selbmann, 1994: 95). En segundo lugar, Selbmann demuestra que, al liberarse de este concepto, que era la base del género dominante en el sistema literario, se abren nuevas posibilidades narrativas para la novela (ibíd.: 95). Por eso, Opiniones del gato Murr preanuncia una nueva época que ya no estará dominada por la tradición individualista del Bildungsroman, sino por otros géneros (la novela histórica, de época, o del espacio) (ibíd.: 96). ${ }^{7}$

Por otro lado, los análisis de la obra de Hoffmann tendieron a profundizar la división entre las secciones de la novela y a limitar el estudio de la tradición del Bildungsroman a la sección paródica, la autobiografía de Murr. Esta separación se acentúa mediante la postulación de una oposición inexacta y esquemática entre los protagonistas, que podemos ver resumida en esta afirmación de Hoffmeister:

\begin{abstract}
Murr no satiriza solamente a Wilhelm Meister, sino también al 'Bildungsphilister' (filisteo cultivado) de la época, así como a Kreisler, el artista romántico par excellence, que forma en sí mismo un contrapunto con Wilhelm. Mientras que Murr madura y finalmente encuentra su lugar en la sociedad, Kreisler permanece sin cambios, un principio extraño y exótico que, como un volcán salvaje, destruye todas las convenciones sociales a su alrededor. Entre él y la sociedad la comunicación está interrumpida. (2004: 89)
\end{abstract}

Hay aspectos acertados en esta afirmación, pero lo que más nos interesa en este momento es que esta cita expone las dos etiquetas falsas a partir de las cuales la mayor parte de la crítica establece una dicotomía simplificadora entre los protagonistas. En primer lugar, Murr es descripto como un Bildungsphilister. Herman Meyer fue el primero en aplicarle este concepto nietzscheano al personaje:

No está mal llamar a Murr un filisteo o, mejor aún, un filisteo cultivado ante datum, si comprendemos que el filisteísmo intelectual aquí no significa simplemente yermo racionalismo, sino que tiene una naturaleza compuesta. Murr es, al mismo tiempo, un racionalista convencido y un sentimentalista exuberante, y el último componente en particular determina el tono, el énfasis constante y ampuloso de su lenguaje. (Meyer, 1961: 122)

Luego, Jacobs retoma el concepto en su capítulo sobre esta novela ("Un gato literario aparece en el rol del héroe problemático, y al final de la historia de desarrollo se encuentra la figura arrogante, incluso ridícula del filisteo cultivado", 1981: 150), pero desplaza su significado, que antes se aplicaba al discurso citado por el personaje y ahora pasa a describir la culminación de su proceso formativo. Con este sentido, la etiqueta se vuelve un lugar común en los estudios sobre Opiniones

7 Sammons (1991: 28ss.) señala que a fines del siglo XIX el Bildungsroman es canonizado como la forma arquetípica de la novela alemana, consignando al olvido gran parte de la literatura del siglo XIX. Este crítico postula que, en realidad, el Bildungsroman tiene dos breves momentos dominantes: en la época de Goethe y durante el modernismo a principios del siglo XX (ibíd.: 32). En esta periodización, la novela de Hoffmann marcaría el final del primer momento. 
del gato Murr, y contribuye a asentar la visión según la cual Murr concluye su formación y se inserta armónicamente en la sociedad, traicionando sus ideales (Nehring, 1997: 204; Stephan, 2019: 203).

El otro lugar común de la crítica, que se advierte en la cita de Hoffmeister, consiste en considerar a Kreisler como un típico artista romántico. ${ }^{8}$ A nivel de la obra, esta definición funciona como el reverso de la etiqueta de Murr, y vuelve más pronunciada la oposición entre los protagonistas: mientras que el gato cede en sus pretensiones artísticas y se une a la sociedad, el músico no abandona su vocación y, entonces, es expulsado de la vida práctica. A nivel de la serie literaria, esto permite agrupar Opiniones del gato Murr junto a las novelas anti-Meister, ${ }^{9}$ que se oponen al desenlace conciliador entre arte y vida del modelo goetheano. ${ }^{10}$

La definición de Bildungsroman que da Morgenstern en 1819 está muy influida por el ensayo de Blanckenburg Versuch über den Roman (1774) y, como veremos más adelante, por las discusiones contemporáneas sobre la novela. ${ }^{11}$ Según Morgenstern, pues:

Podría llamarse novela de formación, primera y principalmente, por su contenido, porque representa la formación del héroe en su comienzo y continuación hasta un cierto nivel de completitud; pero también, en segundo lugar, porque

8 Por ejemplo: "ist Kreisler ein authentischer romantischer Künstler, der zur Kunst berufen ist und sich ihr kompromißlos ergibt" (Nehring, 1997: 204); "he is basically the disillusioned romantic bitterly torn asunder (zerrissen) by visions of ideality on the one hand and awareness of realities on the other" (McGlathery, 1991: 324); "Tiecks junger Maler Sternbald, die Poeten in Eichendorffs Ahnung und Gegenwart, in Dichter und ihre Gesellen, Mörikes Maler Nolten, Hoffmanns genialischer Musiker Kreisler finden mit ihrer ausgereiften Kunst nicht mehr in die Welt, die sie ihnen vermittelte, zurück; sie finden umgekehrt die ihnen zugängliche Welt zunehmend nur noch in ihrer Kunst, und sie verharren und steigern sich in ihrer Besonderheit, indem sie aus ihrer poetischen Welt halb als Getriebene, halb als stolz Vereinsamte ein Gehause zimmern, das ihre sensible Produktivität vor der banalen und auch grausam erfahrenen Alltagswelt der anderen schützt" (Lämmert, 1993: 421); "It is the truly 'Romantic' form of narrating the life of a truly Romantic hero" (Engel, 2008: 284); "Hoffmann ist in seinem letzten Roman bis an den Punkt vorangeschritten, an dem das gesamte poetische Projekt der Romantik selbst zum zitierfähigen Intertext geworden ist, ohne dass es jedoch damit schon aufgehoben wäre" (Kremer, 2009: 356).

9 Hoffmeister explica que este tipo de novelas se originan en la respuesta de los románticos de Jena a la novela de Goethe, y que poseen estas características: "first, to romanticize the protagonist and his world to the extent that they would become part and parcel of the metaphysical real; second, to depict the surrounding environment not in a mimetic fashion but as already transposed by the spiritual life of an ingenious author; third, the author's caprice plays with the given world and constructs a new one as an antidote to bourgeois society" (Hoffmeister, 2004: 83)

10 Estas novelas anti-Meister de los románticos alemanes podrán considerarse o no Bildungsromane según la definición que se maneje de este género (en particular, sobre los requisitos del desenlace). Así, por ejemplo, para Selbmann, que define el género a partir de la tematización protagónica del concepto histórico de Bildung, sin importar si el final es positivo, negativo o paródico (1994: 32-33), estas novelas son Bildungsromane contra-modélicas (ibíd.: 74). Para Koval, en cambio, el desenlace debe contener la agregación exitosa del héroe a la sociedad (2018: 24Ss), y aquellas novelas que no cumplen este requisito, como las de los románticos, no son novelas de formación sino de algún subgénero afín que puede ser "de malformación, de artista y de deformación" (ibíd.: 26).

11 Fritz Martini (1961) localizó la primera utilización del término Bildungsroman, que se creía acuñado por Dilthey en Das Leben Schleiermachers (1870), en una conferencia de Morgenstern de 1810 (publicada en 1817), y demuestra que es recién en la segunda conferencia de 1819 (publicada en 1820 con el título "Ueber das Wesen des Bildungsromans") cuando se lo define por primera vez en base a criterios estéticos e históricos. A su vez, al igual que en las definiciones posteriores, la novela de formación modélica en esta segunda conferencia es Los años de aprendizaje de Wilhelm Meister, mientras que en la primera lo eran las novelas de Friedrich Maximilian Klinger. 
precisamente a través de esta representación promueve la formación del lector, en mayor medida que cualquier otro tipo de novela. (Morgenstern, "Ueber das Wesen des Bildungsromans", citado en Martini, 1961: 57)

En esta definición primigenia podemos distinguir tres elementos principales: en primer lugar, el enfoque en la formación de un individuo particular que alcanza la conformación de una personalidad, de un carácter acabado; en segundo lugar, la descripción del desarrollo de este protagonista desde un estado inicial hasta la realización final, según un orden cronológico, progresivo y teleológico; en tercer lugar, el propósito moral, la formación del propio lector que es promovida por la lectura. Los dos primeros rasgos se mantienen en las definiciones contemporáneas del género. Manfred Engel (2008), por ejemplo, describe tres características para el Bildungsroman: tiene un protagonista único; este posee una profundidad psicológica que se desarrolla de manera gradual y por etapas; la Bildung (la formación de un carácter, de una personalidad individual) es un tema central en la obra, y no solo es narrada, sino que su concepto aparece discutido. Este último punto es el rasgo distintivo y la pieza clave en las definiciones recientes del género (cfr. Sammons, 1991: 41; Selbmann, 1994: 32; Koval, 2018: 18), pues ofrece un anclaje teórico e histórico en torno al concepto de Bildung, entendido en sentido específico, como se cristalizó en la filosofía alemana de finales del siglo XVIII y, en la literatura, en Los años de aprendizaje de Wilhelm Meister. Lo que es relegado en definiciones como la de Engel (y en la mayoría de las definiciones contemporáneas) es la particular intención estética del género: el tercer rasgo de la definición de Morgenstern. Recuperar este aspecto nos permitirá reconocer el lugar del Bildungsroman en el sistema literario del Kunstperiode; a partir de esto, podremos comprender el desafío estético que supone la novela de Hoffmann y por qué podemos afirmar que marca el final de una etapa de la evolución de la novela alemana. Para entender el peso fundamental asignado al efecto de la lectura en la definición de Morgenstern debemos atender a las particulares condiciones del mercado literario de la época y las discusiones sobre la novela.

Cabe recordar, en primer lugar, que Morgenstern fue un pionero incógnito en la utilización del término Bildungsroman, y que esta categoría recién fue popularizada para la crítica literaria por Wilhelm Dilthey. ${ }^{12}$ Hoffmann jamás pudo haber conocido la definición del profesor de Dorpat, que no tuvo ninguna repercusión en las discusiones sobre literatura de la época. Lo que nosotros llamamos Bildungsroman, y consideramos como un género literario de la prosa narrativa moderna entre muchos otros, equivalía entonces prácticamente a la novela o, más precisamente, a la novela culta. Esto se comprueba, por ejemplo, en la siguiente definición sobre la novela (Roman), en el artículo correspondiente de la enciclopedia Brockhaus de 1817:

12 Primero emplea el término Bildungsroman en Das Leben Schleiermachers y luego, da la definición clásica en Vida y poesía (1905): "[las novelas de formación] nos representan al joven de aquellos días; nos exponen cómo entra en la vida bajo un dichoso amanecer, cómo busca las almas afines a la suya, cómo se encuentra con la amistad y con el amor, y cómo choca y lucha con las duras realidades del mundo y va haciéndose hombre bajo las múltiples experiencias de la vida, cómo se encuentra a sí mismo y cobra la conciencia de su misión en el mundo" (2016: 325). 
La historia de la formación individual, la vida y el destino de un individuo desde su nacimiento hasta su formación completa, pero sobre y con la cual se deduce todo el árbol de la humanidad según sus diversas ramificaciones en el hermoso período de su maduración y perfeccionamiento, los años de aprendizaje del discípulo hasta que se eleva a maestro: eso es la novela (Conversations-Lexiconoder encyclopädisches Handwörterbuch für gebildeteStände, citado en Vollmer, 1993: 25).

Sin embargo, es evidente que esta definición excluye a muchas obras que también consideraríamos novelas (esto lo evita Morgenstern al utilizar un término más acotado), y debe entenderse como una toma de posición muy difundida en un debate generalizado en la época a partir del crecimiento del mercado literario.

Reinhard Wittmann (2011) sostiene que en 1800 es posible hablar de una revolución de la lectura, tanto por los cambios cuantitativos como por los cualitativos que se producen respecto a las décadas previas. En los territorios de lengua alemana, el público lector (especialmente la burguesía) aumenta del 5\% de la población en 1700 al $30 \%$ en 1800 . Esto se debe a una multitud de factores, como la expansión del sistema educativo, el surgimiento de instituciones que promueven la lectura (bibliotecas de préstamo, sociedades literarias), el reemplazo del latín por el alemán como idioma principal en los libros impresos (en 1700 el 38\% de las publicaciones estaban en latín, en 1800 el 4\%). Al mismo tiempo, se desarrollan nuevos hábitos de lectura (se pasa de una lectura intensiva a una extensiva), se producen cambios en el gusto del público y, por lo tanto, en la oferta de libros. Por ejemplo, los libros bajo la rúbrica de belles lettres pasan de ocupar el sexto lugar de la oferta en el año 1700 (solo el 6\% de los libros impresos, de los cuales la mayoría son libros religiosos y de teología) a estar en el primer puesto en 1800 ( $21,45 \%$ del total) y, dentro de este grupo, el mayor crecimiento lo experimenta la novela (pasa de representar el 2,6\% de los libros impresos en 1740, al 11,7\% en 1800) (Schön, 2001; Brandes, 2005; Wittmann, 2011).

Una de las consecuencias de un cambio histórico-social como este para la obra de Hoffmann es expuesta por Meyer en el ensayo que ya citamos. Este crítico compara el discurso citado en la obra de Wieland, a mediados del siglo XVIII, y el de la obra de Hoffmann, a principios de siglo XIX, y expone los límites y las posibilidades de cada uno en relación con su contexto. Mientras que el primero debía recurrir al arsenal de citas de la Antigüedad clásica y a las belles lettres extranjeras para darle un gusto a un grupo de eruditos, el segundo, en cambio, se encuentra al final de un gran período de florecimiento literario, y puede recurrir a una amplia reserva de literatura alemana canonizada, cuyas forma y estilo se han asentado en la memoria de un público lector amplio (Meyer, 1961:117). Esto, podemos agregar, es una condición de posibilidad para escribir una parodia como Opiniones del gato Murr.

En los primeros años de la época de la Restauración, la novela, en particular, alcanza una producción y un consumo masivos (es el género más popular y tiene una extensa variedad de subgéneros), y se la compara con un diluvio que inunda el mercado literario (Vollmer, 1993: 25). Entre 1815 y 1820, solo seis autores (Julius 
von Voß, Friedrich Laun, Heinrich Müller, Carl Nicolai, Christoph Hildebrandt y Gustav Schilling) publican 94 novelas. Como es previsible, paralelamente, crecen las discusiones teóricas sobre el género ${ }^{13}$ y su condena moral. La sentencia que declara a la novela como una forma anti-poética, resume Vollmer, se sustenta en que, por un lado, la "masa novelística" se aparta de los objetivos e ideales instructivos para la formación y edificación de las personas y, por otro lado, el "entretenimiento vulgar" hace que la satisfacción de los deseos físicos sea lo principal en la escritura (ibíd.: 23-24).

Sobre este conjunto de novelas de producción masiva, se destaca una forma positiva del género, culta y prestigiosa, que a diferencia del mero entretenimiento, tiene un efecto provechoso sobre el lector. Como afirma Vosskamp (1992), la Bildung funciona como esa marca de prestigio y, al mismo tiempo que legitima a la novela como un tipo de literatura seria, le impone una estructura narrativa (el desarrollo cronológico y progresivo del protagonista) que debe satisfacer su intención (su justificación) estética (la formación del lector).

En la sección de Murr se reconocen estas características específicas del género, pero aparecen despojadas de su función original elevada, seria, y se las ridiculiza. En su autobiografía, Murr enfatiza desde el comienzo su pretensión de influir positivamente en el lector a partir de la exposición de su formación, su recorrido ordenado hacia la definición de su carácter. Ya en el prólogo, el narrador anuncia su propósito aleccionador, su base didáctica: "Con la seguridad y tranquilidad que son innatas al verdadero genio entrego al mundo mi biografía, para que aprenda cómo se educa uno para ser un gran gato [zum großen Kater bildet]" (129). Y más adelante: "Pues es sin duda curiosísimo e instructivo que una gran inteligencia se explaye minuciosamente en una autobiografía sobre todo lo que le ocurrió en su juventud, por insignificante que pueda parecer" (151). Pero, a lo largo de toda su narración, se revela que sus elevadas pretensiones contrastan irónicamente con la banalidad de su experiencia. Como dice Steinecke: "La formación persigue aquí siempre fines concretos, ventajas palpables, la estima de los demás, una vida mejor y más cómoda" (1992: 950). Con esto se resumen los principales procedimientos con los que Hoffmann parodia el género en esta sección: la repetición, la hipérbole y la ironía. Esto se repite, como veremos a continuación, en la prosecución ad absurdum (Mahonney, 1988: 51; Nehring, 1997: 201) de la estructura narrativa básica del género, en la manera en que Murr presenta su vida como una serie de acontecimientos significativos que se suceden lógica y progresivamente hacia un objetivo último, la realización de su personalidad.

En primer lugar, el narrador ordena su vida mediante la descripción de etapas o episodios bien delimitados y ordenados cronológicamente. Esto se ve tanto en los nombres de los capítulos ("Los meses de la juventud", "Experiencias juveniles",

13 En el primer prólogo de Murr puede leerse una referencia a esta situación. Cuando Murr menciona el "severo juicio de la crítica" (128), se refiere a un hecho reciente, el creciente número de revistas que reseñan obras e influyen en decisiones editoriales y, de manera relativa, en el gusto del público. Murr es consciente de esta distancia entre crítica y público general cuando sostiene que él no escribe para los "insensibles reseñadores" sino para las "almas sensibles", los "ánimos puros e infantiles" (íd.). 
"Meses de aprendizaje [Lehrmonate]", “Los meses maduros del varón"), en donde la alusión paródica a la novela de Goethe es inconfundible, como en los comentarios de Murr, que dividen su biografía en episodios (nacimiento, período de poética melancolía, edad del pavo, salida al mundo, enamoramiento, etc.) separados por "ritos de paso". ${ }^{14}$ En segundo lugar, Murr explicita que su vida se desarrolla de manera progresiva en la dirección de la conformación de su carácter: "Nada trastorna mi formación [Bildung], nada se opone a mis inclinaciones, avanzo con pasos de gigante hacia la plenitud" (184). Además, el narrador precisa cuál es el objetivo final de su desarrollo: su meta específica es la agregación a la sociedad. Con el tono hiperbólico que lo caracteriza, Murr revela su confianza en la teleología positiva de su vida desde el comienzo, cuando, después de describir su nacimiento en uno de los primeros párrafos, concluye: "avanzaba a pasos de gigante en mi educación para el mundo [in der Bildung für die Welt]” (135).

Sin embargo, a pesar de lo que constituye un lugar común en los análisis de la novela, en ningún momento se realiza la agregación a la sociedad. ${ }^{15}$ Murr describe tres experiencias de socialización o salidas al mundo, las tres terminan de manera fracasada y acentúan su aislamiento. Su primera salida ocurre por accidente, cuando la berlina inglesa en la que descansaba en el patio de la casa comienza a rodar, y Murr se despierta en una ciudad caótica como "un recién llegado sin experiencia” (224). Las burlas de la gente, la dificultad para conseguir alimento, la falta de comodidad a la que está acostumbrado lo llevan a rechazar la vida social, y decide volver a recluirse. El segundo intento ocurre bajo la guía del gato Muzius. Los guías o maestros son personajes secundarios típicos del Bildungsroman y su función consiste en favorecer la maduración del protagonista (Koval, 2018: 28). Murr tiene varios maestros (Abraham, su madre Mina, Skaramuz), pero los más importantes e influyentes son Muzius y el perro Ponto. Como en el modelo goetheano, estos personajes intervienen para orientar a Murr hacia la vida práctica o en sociedad. Muzius, por ejemplo, obliga al protagonista a dejar la comodidad del hogar y lo introduce en una hermandad de gatos (con la que Hoffmann alude a las asociaciones estudiantiles de la época). La expectativa con la que Murr se incorpora a este grupo contrasta irónicamente con la superficialidad de sus actividades (que se limitan, básicamente, a comer, beber y cantar). Esta experiencia finaliza cuando, al ser tomados por alborotadores, la hermandad es perseguida y debe disolverse, y Murr vuelve a la soledad de su hogar. El último episodio de su vida que narra el protagonista es su tercer intento de agregación a la sociedad: “ahora que había llegado a meses más maduros y recopilado suficiente experiencia de la vida, ya no podía encontrarme con aquellos peligros en los que había caído cuando el destino me arrojó, joven menor de edad, al mundo" (457). Murr reconoce al perro Ponto y su nuevo amo, el barón

14 Por ejemplo: "[una circunstancia] hizo una cesura en mi vida" (164), "[un incidente] marca el paso a los años de formación madura" (183); "Con ello pude poner fin al primer período de mi vida, y pasar a los meses juveniles propiamente dichos" (202); "Considero este funeral el punto de inflexión que puso fin a mis meses de aprendizaje" (441). Cfr. Koval (2018: 21) para la aplicación de la categoría "ritos de paso" al análisis literario de este tipo de novelas.

15 Esto lo explicita además la postdata del editor, donde se informa la muerte de Murr: "Así vuelve a darse una prueba de que el genio temprano no siempre progresa; o se hunde en un anticlímax hasta la indiferencia sin carácter ni espíritu y se pierde entre la masa, o no llega a cumplir muchos años..." (523). 
Alzibiades von Wipp, como los modelos de formación superior orientada a la vida práctica, y decide someterse a la instrucción del primero. Guiado por Ponto, Murr ingresa en un círculo de perros elegantes al que parece adaptarse, renunciando a sus altos ideales, pero pronto reconoce la ignorancia y la superficialidad de este medio, se arrepiente y decide volver a recluirse.

De este modo, aunque Murr sea un héroe ridiculizado, el hecho de que no logre incorporarse a ningún grupo social, a pesar de sus reiterados intentos, le reserva la posibilidad de criticar a su entorno y, particularmente, a su principal maestro, el perro Ponto. La función de este personaje y su relación con el Bildungsheld replica de manera degradada la del personaje de Jarno con Wilhelm en los Años de aprendizaje. Como este, Ponto critica la vida contemplativa de Murr y se encarga de promover sus habilidades prácticas. El narrador reconoce que su maestro consigue obtener resultados materiales, pero también señala que en esa conducta hay "algo contra lo que mis sentidos íntimos e innatos se rebelan. Una cierta lisonja sumisa, una negación de la propia conciencia de la noble naturaleza” (237). En el segundo volumen, la crítica al maestro se refuerza cuando Ponto expone cómo su actitud con su nuevo amo le trae en realidad diversos beneficios (una vida con todas las comodidades, lujos, alimentos selectos, juegos, y carente de exigencias), y aparece ante los ojos de Murr como un hipócrita servil y oportunista.

Todo esto demuestra que no puede sostenerse la afirmación sobre la rendición o la agregación de Murr a la realidad concreta. El narrador es consciente de esta falta a su objetivo, y debe justificarla exaltando su individualidad y postulando una diferencia irreconciliable con su entorno: “¿acaso no tengo que ver por donde mire que estoy solo, como en el más profundo desierto, porque no pertenezco a esta época, sino a una futura de superior formación, ya que no hay un alma que sepa admirarme como es debido?" (293). Así es como esta sección de la novela incorpora la variante romántica del Bildungsheld.

Como señala Martin Swales (1991), desde las Lecciones sobre estética de Hegel, el género del Bildungsroman se estructura a partir de la descripción de un conflicto fundamental. Para Hegel, el conflicto se da entre la poesía del corazón y la prosa de las circunstancias externas (1987: 786), para Lukács, entre el ideal y la concreta realidad social (2002: 116), ${ }^{16}$ para Swales, entre la potencialidad individual (el Nebeneinander) y la realidad práctica (el Nacheinander) (1991: 52). La interpretación clásica señala que, por un lado, Los años de aprendizaje de Wilhelm Meister representa el punto medio deseable entre los dos polos, ${ }^{17}$ en tanto el protagonista logra conciliar su vocación personal y la vida práctica al integrarse felizmente en una comunidad plena de sentido (Koval, 2018: 174). Por otro lado, se afirma que las novelas de los escritores románticos alemanes que se oponen al modelo goetheano (el ejemplo prototípico de este grupo es la novela inconclusa de

$16 \mathrm{Ni}$ Hegel ni Lukács utilizan el término Bildungsroman. Sobre la correspondencia entre novela de formación y novela en la definición de Hegel véase Selbmann (1994: 12ss). Lukács, en Teoría de la novela, utiliza la denominación "Erziehungsroman" (novela pedagógica o de educación) en su análisis sobre Los años de aprendizaje de Wilhelm Meister.

17 Existe, sin embargo, una corriente de interpretaciones que ponen en duda el equilibrio final de la novela de Goethe (cfr. Swales, 1991; Engel, 2008). 
Novalis, Enrique de Ofterdingen, de 1802) rechazan la realidad concreta y exaltan el principio poético o individualista. ${ }^{18}$ Lukács (2002) llama a este camino la vía contemplativa o cierre en la interioridad, ${ }^{19}$ para Engel (2008) esta es la variante trascendental del Bildungsroman; para Hoffmeister (2004) y muchos otros estas son las novelas anti-Meister. ${ }^{20}$

Como ya adelantamos, en la sección autobiográfica de Opiniones del gato Murr, la imposibilidad de exponer una socialización exitosa le impide al narrador realizar su objetivo específico (agregación a la vida práctica), y su carácter acabado, entonces, debe asentarse sobre otra base (el otro polo de oscilación del género): la excepcionalidad de su personalidad individual. Esto se observa en los siguientes ejemplos:

Si me entrego a otros trabajos literarios, si escribo por ejemplo críticas, que me corresponden porque ante todo soy poeta, escritor, artista, floto, superior a mí mismo, como modelo por supuesto inalcanzable, como ideal de la perfección, y por tanto el único capaz de emitir una sentencia competente; ¿quién podrá alcanzar mi punto de vista, quién compartir conmigo mis criterios? [...] penetró en mi alma una desolada indiferencia frente a todo bien terreno [...] Una cierta melancolía, como la que suele atacar a los jóvenes románticos cuando superan la lucha de los grandes y sublimes pensamientos en su interior, me empujó a la soledad. $(293,377,178)$

Aquí, nuevamente, la intención paródica se construye mediante la combinación de la repetición, la hipérbole, y el contraste irónico entre la retórica elevada y los acontecimientos triviales. Esto último se vuelve evidente si reponemos el contexto de las últimas dos citas, en las que el protagonista, respectivamente, resume las consecuencias de una resaca después de una noche de celebración con los gatos de la hermandad, y describe sus sentimientos tras comerse una cabeza de arenque que había prometido compartir con su madre.

En resumen, el alcance de la parodia en la sección de Murr revela la posición de la novela de Hoffmann en la serie literaria: el agotamiento del Bildungsroman se refleja en la adopción paródica de las dos variantes arquetípicas del género, la aspiración a una síntesis con la realidad y la exaltación romántica del individuo. En la sección de Kreisler, veremos a continuación, el protagonista también se encuentra ante la disyuntiva de la agregación a la sociedad o la reclusión, pero, a la inversa de Murr, rechaza las dos opciones.

18 Las dos variantes están implícitas ya en la definición de Dilthey: "El tema de Goethe era la historia de un hombre que se va formando para la acción, el de los dos románticos [el Sternbald de Tieck, el Ofterdingen de Novalis] era el poeta; el héroe de Hölderlin es la naturaleza heroica que pugna por manifestarse en el todo y que, por último, se ve obligado a replegarse dentro de su propio pensamiento y su poesía" (Dilthey, 2016: 352).

19 Para Lukács, el peligro en el que incurre Enrique de Ofterdingen de Novalis es "el peligro de romantizar la realidad hasta trasponerlo todo en lo meta-real, o, cosa que muestra más claramente el peligro propiamente dicho, el artístico, en una esfera totalmente desprovista de problemas" (2002: 122).

$20 \mathrm{Cfr}$. supra. 
La relación de la novela de Hoffmann con la tradición del Bildungsroman apenas ha sido estudiada en la parte de Kreisler. Los puntos comúnmente analizados se limitan a los rasgos formales de la novela y al (mal entendido) desenlace del protagonista. En primer lugar, se señala que, mientras que la novela de formación se caracteriza por presentar el desarrollo de un personaje de manera cronológica y progresiva, la biografía de Kreisler está incompleta, se presenta de manera fragmentada y desordenada, y no tiene lugar ninguna formación en el protagonista (Steinecke, 1992: 951; Selbmann, 1994: 75; Kremer, 2009: 355). Esto puede resumirse con las categorías de Tomashevski (2011:302) diciendo que, a diferencia de los protagonistas de los Bildungsromane, que como Murr, son caracteres cambiantes, Kreisler es un carácter constante. Un aspecto relacionado es el tema de la circularidad: frente a la forma lineal de la novela de formación, la biografía de Kreisler se caracteriza por ser circular, ya que el último fragmento (en el que Kreisler recibe la invitación de Abraham para regresar a Sieghartsweiler y el anuncio de la próxima fiesta de cumpleaños de la princesa madre) antecede al primero (donde se narran los sucesos de la fiesta) (Kremer, 2009: 346). Además, este rasgo está reflejado en el nombre del protagonista (Kreis significa círculo en alemán); sentido que él mismo comenta en un diálogo con Benzon (cfr. 186). Como señala Segebrecht (1967: 75), a nivel onomástico, esto constituye una oposición contra el significado lineal original del género, simbolizado en el apellido de Anton Reiser (Reise, en alemán, significa viaje), el protagonista de la novela de Karl Philipp Moritz que se suele considerar un antecedente del Bildungsroman. Por último, como vimos en la introducción, el mayor lugar común en los estudios sobre esta sección consiste en afirmar que Kreisler, en falsa oposición con Murr y en falsa asociación con los protagonistas de las novelas anti-Meister, es el típico personaje romántico que le da la espalda a la sociedad para vivir dedicado a su vocación personal. Creemos que la relación de esta sección de la novela con el Bildungsroman no se agota en aquellos rasgos formales, sino que también se encuentra en el contenido de la obra. Veremos que los tópicos centrales del género que, en la parte de Murr, son asumidos acríticamente por el protagonista y parodiados, en particular, el énfasis individualista y la organización progresiva y teleológica de la vida, son atributos expuestos en personajes secundarios, discutidos en los diálogos y manifiestamente rechazados por Kreisler.

La crítica al individualismo se dirige contra una de sus principales fuentes: Rousseau, quien popularizó un estilo centrado en el desarrollo de la personalidad y su autoconocimiento (Segebrecht, 1967: 64), y tuvo una influencia central en la literatura alemana del período artístico (Jauss, 1995: 75ss.), en particular, en la noción de Bildung (Koval, 2018: 37ss.). En un importante diálogo con Abraham y un consejero privado anónimo, al final del primer capítulo, Kreisler recuerda un episodio de su infancia en el que, imitando al narrador de las Confesiones, pretende acostarse en la cama y "recibir una ópera en espíritu" (215), pero no solo no lo consigue, sino que se queda dormido, no nota que la casa se incendia y el tío lo castiga. Como señala Segebrecht, aquí, Kreisler demuestra que el entusiasmo en sí mismo no promueve ninguna producción artística, y que los esfuerzos 
por auto-conocerse "a la manera de Rousseau" son inútiles para él (Segebrecht, 1967:66). Si comparamos las alusiones a Rousseau por parte de Murr y por parte de Kreisler, respectivamente, hallaremos sintetizadas las dos formas en que esta novela rechaza la tradición de la individualidad excepcional: la adopción paródica y la exposición para su negación. Mientras que Kreisler sostiene que el modelo rousseauniano no puede aplicarse a su persona, en la sección autobiográfica, esta tradición se reconoce como un modelo a seguir. Murr, después de referirse a otro episodio de las Confesiones, dice: "Yo me encuentro ahora en el mismo caso que aquel venerado autobiógrafo” (501).

Este procedimiento se repite a propósito de la organización temporal del relato. En el diálogo recién mencionado, el consejero privado representa el papel del lector de novelas de formación, e intenta explicar la personalidad de Kreisler mediante su biografía. En primer lugar, el consejero le reprocha al músico que no quiera compartir la historia de su vida (205), y le solicita que dé información sobre su pasado para poder explicar su personalidad actual a partir de la reconstrucción de una serie de etapas ordenadas lógicamente. En segundo lugar, intenta localizar en la biografía de Kreisler un conflicto entre su vocación y el mandato paterno (cuyo lugar ocupa el tío), lo que constituye un motivo más específicamente propio del Bildungsroman (cfr. Koval, 2018: 25). La réplica de Kreisler, de nuevo, niega ese rasgo estructural del género (enfatizado por Murr), según el cual la personalidad se conforma a partir de una secuencia de episodios significativos:

mientras esperabas quizá lo extraordinario, sólo puedo servirte lo vulgar, tal como se repite mil veces en la vida. Así, es cierto que no fue coacción educativa, ni especial terquedad del destino, sino el más habitual curso de las cosas, el que me empujó a ir involuntariamente allá donde yo no quería ir. (218)

El mismo rasgo, además, es rechazado mediante su presentación negativa en un personaje secundario. Es ilustrativo de la escasa atención que ha recibido esta parte de la novela el hecho de que los estudios sobre la relación de Opiniones del gato Murr y el Bildungsroman no hayan reparado en la historia de Abraham, el misterioso prestidigitador de la corte de Sieghartsweiler. Esta historia se describe en una analepsis en el segundo volumen, según el modelo de una típica Bildungsgeschichte, y recuerda a la situación inicial de Wilhelm Meister, Joseph Berglinger ${ }^{21}$ o Enrique de Ofterdingen. El joven Abraham es obligado por su padre a trabajar de manera repetitiva en el oficio familiar, la construcción de órganos. La disciplina que impone el padre choca con los sentimientos del hijo; el narrador resume la situación de esta manera: "Exactitud, perseverancia, trabajo bien hecho lo eran todo para el anciano; no tenía sentido alguno del espíritu, del sonido" (370). Como en el caso de los protagonistas de Goethe y Novalis, la posibilidad de evadirse de esta imposición paterna se presenta con un viaje relacionado con las actividades de su gremio. También como en esos ejemplos,

21 El protagonista del último relato de Efluvios cordiales de un monje amante del arte (1796), de Wilhelm Heinrich Wackenroder. El maestro de capilla Berglinger es el antecedente literario más claro del personaje de Kreisler, y la comparación entre ambos expone de manera condensada las diferencias que existen entre la poética hoffmanniana y la del Romanticismo temprano. 
durante el viaje, Abraham reconoce y desarrolla su vocación personal, su faceta individualista: se destaca por sobre la masa inculta de viajeros, se acentúan su interés artístico y el rechazo de las convenciones familiares, se enamora de una joven extraña, misteriosa, frágil (370). De este modo, en la novela de Hoffmann se establece una oposición con un sentido generacional entre el viejo maestro, que pertenece a una época anterior y cuya vida puede describirse en los términos de una Bildungsgeschichte, y el joven protagonista, que ve ese esquema como un modelo anticuado e inválido.

La descripción de Abraham como una persona perteneciente a una época anterior que no logra adaptarse y hallar la felicidad en los nuevos tiempos se encuentra muy desarrollada en la novela. Este prestidigitador era la figura central de la vieja corte de Sieghartsweiler, en una era donde eran aceptados los prodigios; tenía una relación cercana con el viejo príncipe, quien, maravillado por los artilugios del ilusionista, lo había llevado a vivir a su propio palacio, en unas estancias conectadas con su estudio. Pero, con la muerte del viejo príncipe y la invasión napoleónica, comienza una nueva época que, según un esquema temporal habitual en Hoffmann, se caracteriza por ser excesivamente racionalista, superficial y enemiga de lo maravilloso. ${ }^{22}$ Con el nuevo gobierno del príncipe heredero Irenäus, Abraham pasa a ocupar un lugar periférico (simbolizado espacialmente en su traslado a la cabaña del bosque de las afueras), y el centro de la escena palaciega es dominado por su antagonista, la consejera Benzon, a quien él reconoce como "el verdadero astro brillante que todo lo ilumina aquí [en la corte]" (344) (con una metáfora crítica de la Ilustración, recurrente en Hoffmann). Si bien Benzon, como funcionaria intrigante y materialista, es el personaje más negativo de la novela, en un diálogo con Abraham esboza una crítica al individualismo consecuente con la postura del autor en toda su obra: “ ¿Es que no crees, anciano, que también habrás sufrido alguna desdicha, que es un peligroso juego elevarse sobre esas convenciones y querer acercarse más al espíritu del mundo mixtificando el propio ser?" (349). Más adelante, Abraham parece darle la razón a Benzon, cuando se cuestiona haber elegido una vida solitaria: " $i Q u e ́$ te importa [...] el mundo entero del que te burlaste porque lo tomaste por loco cuando tú eras el más loco! En tu trabajo, en tu trabajo tenías que quedarte, construir órganos, y no jugar a brujo y a vidente" (476). La diferencia generacional y en los modelos formativos de Kreisler y Abraham, y la crítica a la excentricidad y el aislamiento de este personaje son una primera razón para cuestionar aquel lugar común de la crítica que afirma el carácter anti-Meister de la novela. Pero aún hay más argumentos.

Es una característica de la obra de Hoffmann exponer la realidad de la nobleza y la burguesía alemanas contemporáneas en modo satírico (Mayer, 1959: 210). Esto se observa en Opiniones del gato Murr, especialmente, en los personajes de la corte de Sieghartsweiler. El excesivo y ridículo interés material, la intriga política, el arribismo, la ignorancia y la falta de sentido artístico que caracterizan

22 En Klein Zaches, por ejemplo, observamos el mismo esquema en la oposición entre el gobierno del príncipe Demetrius, que les permite a los seres maravillosos habitar en su territorio, y el de su sucesor, el príncipe Paphnutius, que expulsa a las hadas e introduce la Ilustración (Hoffmann, 1985: 543). 
a esta corte ficticia no ofrecen ninguna posibilidad de reconciliación para el protagonista. Sin embargo, es significativo que los personajes o espacios más ajenos al mundo cortesano no ofrezcan una alternativa positiva. Al ejemplo de Abraham se suman la historia del pintor Ettlinger y el episodio en la abadía de Kanzheim, que representan dos posiciones extremadamente opuestas al espacio cortesano y, también, dos modelos igualmente rechazados por el protagonista. Hedwiga le cuenta a Kreisler cómo al verlo por primera vez en el parque lo confundió con Ettlinger, un viejo pintor de la corte que se había enamorado de la princesa madre y, enloquecido, fue encadenado en el castillo. Por un lado, varios elementos, además de la comparación de la princesa, aproximan al protagonista a este personaje: ambos son artistas de la corte, Kreisler se ve reflejado como Ettlinger en el lago y lo llama su doble (278), y también admite que "[s]iempre había tenido la idea fija de que la locura le acechaba” (271). Pero, por otro lado, el músico se opone al modelo de artista exaltado hasta la locura que representa el pintor: dice que este no pertenece a los verdaderos artistas, que "ni perecen miserablemente de tisis ni se vuelven locos” (272). Además, Kreisler afirma que su conducta serena está lejos del tipo exaltado, de la "triste locura del estudiante" (364), y lo mismo dice Abraham: "el señor maestro de capilla es, ahora como siempre, un hombre razonable y tranquilo, y no el fantasioso o loco por el que muchos quisieran tenerlo" (211). Los referentes tácitos al final de esta cita (que podría aplicarse a los críticos que ven en Kreisler un ejemplo más de artista romántico) son los personajes de la corte, y Abraham devela así el interés de aquel grupo por desestimar, mediante la acusación de locura, la crítica social que expone la ironía kreisleriana. ${ }^{23}$

En adición a esto, la diferencia entre Kreisler y el prototipo del artista romántico se evidencia en su rechazo al aislamiento y su permanencia en el ámbito de la corte. Este es el tema principal del episodio en la abadía de Kanzheim, que ocupa la mayor parte de las hojas de maculatura del segundo volumen. El entorno de la abadía es descripto como un "paraíso" (388), un "asilo" y una "isla" (391), donde los monjes y el abad Chrisostomus viven en armoniosa comunidad. Allí, en soledad, Kreisler puede componer piezas religiosas sin esfuerzo, "en una alta y espaciosa estancia del edificio principal” (368). El abad le propone al músico que renuncie al mundo e ingrese en la orden para vivir exclusivamente dedicado al arte (392). Chrisostomus interpreta la vida del protagonista de esta sección según el esquema del Bildungsheld en el que la vida es un proceso de búsqueda; en particular, según el modelo anti-Meister, que no concluye con la agregación a la sociedad, y le ofrece un desenlace a su medida (la reclusión):

Me refiero a aquellos que son y no dejan de ser extraños en el mundo, porque pertenecen a una existencia superior y consideran las exigencias de esa existencia superior condición de la vida, pero, persiguiendo sin descanso lo que no pueden encontrar en este mundo, eternamente sedientos de un ansia que no puede satisfacerse nunca, van de acá para allá buscando inútilmente paz y tranquilidad [...] Sólo la soledad, una vida uniforme sin hostiles interrupciones,

23 Benzon la define así: "con esa fantástica afectación, con esa cortante ironía, no creáis nada más que inquietud, confusión, total disonancia en todas las relaciones convencionales existentes" (185). 
y sobre todo la siempre despejada visión del mundo de la luz al que pertenecen, puede establecer el equilibrio y hacerles sentir en su interior una satisfacción supraterrena que no pueden conseguir en el confuso trajín del mundo. Y vos... vos, Johannes, sois uno de esos hombres a los que el poder eterno eleva a lo celeste bajo la presión de lo terreno. (393)

Vemos aquí nuevamente el punto de vista de otro personaje que analiza e intenta determinar la vida del protagonista (el consejero anónimo indagaba en su pasado, el abad en su futuro). Pero Kreisler se niega a aceptar este desenlace, que implica un tipo de resolución evasiva o contemplativa del conflicto y, como sabemos por el primer capítulo, regresa a la corte. Este rechazo a la evasión que propone el abad se replica en la oposición de dos filosofías de la historia. Mientras que Chrisostomus postula una "diferencia entre los antiguos y devotos tiempos y los actuales y corrompidos" (446), el músico cree que la "virtud no había desaparecido en modo alguno de la faz de la Tierra” (448). De este modo, el presente es rehabilitado para la narración, y su descripción satírica impide hablar de una aceptación aproblemática. Finalmente, el último episodio que se narra de la vida de Kreisler (y de la novela) refuerza la crítica a la evasión del presente. Esto se ve en el enfrentamiento entre el padre Cyprianus, caracterizado por su severidad, ascetismo y desprecio por la vida terrena, y el protagonista, que lo confronta con las siguientes palabras: “¿Y quién eres tú, monje loco, para querer elevarte por encima de todo lo humano?” (513).

La oposición de Kreisler a este personaje está en la línea de su diferenciación respecto a Ettlinger y a Abraham y, en otro nivel, escenifica su alejamiento del modelo típico de artista romántico. Basta contrastarlo con un solo ejemplo representativo de esa tradición, el personaje de Julius en la novela anti-Meister Lucinde (1799), de Friedrich Schlegel:

[Julius] Rompió todos los lazos anteriores y se hizo de repente independiente por completo. Consagró su fuerza y su juventud al excelso trabajo y entusiasmo artísticos. Olvidó su época y se formó según los héroes del pasado, cuyas ruinas amaba con adoración. Para él mismo tampoco había presente, pues vivía sólo en el futuro y en la esperanza de consumar alguna vez una obra eterna como monumento de su virtud y de su dignidad. (Schlegel, 1987: 65)

Aquí encontramos, por un lado, el hipotexto serio del estilo de Murr y, por otro lado, la actitud opuesta a la que describimos en Kreisler. El resumen de los posicionamientos de este personaje en la abadía de Kanzheim -su rechazo al aislamiento en armonía con la naturaleza, a la idealización del pasado y la condena del presente, a la afirmación de la superioridad individualista- niega punto por punto las características del héroe anti-Meister.

En conclusión, las dos secciones de Opiniones del gato Murr dialogan centralmente con la tradición del Bildungsroman. La autobiografía de Murr emplea la estructura básica del género al mismo tiempo que la despoja de toda su seriedad y dignidad canónica. La intención didáctica, el ordenamiento cronológico de los acontecimientos, delimitados en etapas de desarrollo progresivo, la guía de los 
maestros, la explicitación de los objetivos, la voluntad de formarse para la vida práctica y la discusión sobre su formación: todos estos elementos del Bildungsroman son repetidos, exagerados y contrastados irónicamente con una historia banal. El recorrido de Murr por el camino ideal del Bildungsheld se interrumpe en un punto crucial: en el desenlace. La imposibilidad de agregarse a la sociedad, además de reservarle la mirada crítica del outsider, obliga al narrador a redirigir la teleología de su formación hacia el interior. Entonces, la parodia abarca ahora también al otro polo tradicional del género, el poético, el de la variante antiMeister. Es una marca de cohesión y unidad de la novela de Hoffmann que los mismos rasgos criticados por Murr reaparezcan en la sección de Kreisler. Vimos que aquellos elementos del género que uno proseguía ad absurdum son expuestos y rechazados explícitamente por el otro. La linealidad, la división en etapas cronológicas, la progresión de la formación del protagonista son negados en la sección de Kreisler, en la forma y en el contenido. La excentricidad, la soledad, la formación exclusivamente artística, todos estos rasgos del artista romántico se le ofrecen al protagonista (como desarrollos posibles, como dobles en personajes secundarios), y son invariablemente rechazados. Kreisler no se agrega felizmente a la prosa de la corte, pero también rechaza el idilio individualista de la abadía.

Opiniones del gato Murr, al parodiar la estructura fundamental del Bildungsroman, y al rechazar los modelos de formación clásicos (para la vida práctica o para la poesía), se opone tanto a la variante goetheana como a la romántica del género, por lo que no debe ubicársela junto a las novelas anti-Meister. En adición a esto, si ahora recordamos el lugar dominante del Bildungsroman en el sistema literario alemán de la época, podemos sostener que la obra de Hoffmann no solo señala el agotamiento del género, sino que anuncia un cambio estético más general: la novela alemana, que a fines del siglo XVIII, asociada a la Bildung, postula su dignidad artística, casi medio siglo después empieza a desprenderse de su excusa pragmática para afirmar su autonomía. 


\section{Bibliografía}

"Brandes, H. (2005). The Literary Marketplace and the Journal, Medium of the Enlightenment. En Becker-Cantarino, B. (ed.). German Literature of the Eighteenth Century. The Enlightenment and Sensibility, pp. 79-102. Vol. 5 de The Camden House History of German Literature. Nueva York: Camden House.

" Dilthey, W. (2016). Vida y poesía. Trad. de W. Roces. México: FCE.

"Engel, M. (2008). Variants of the Romantic 'Bildungsroman' (with a short note on the 'artist novel'). En Gillespie, G.; Engel, M.; Dieterle, B. (eds.). Romantic prose fiction, pp. 263-295. Vol. XXIII de A Comparative History of Literatures in European Languages. Amsterdam/Philadelphia: John Benjamins.

" Hoffmann, E.T.A. (2008). Opiniones del gato Murr. Trad. de C. Fortea. Madrid: Cátedra.

"Hoffmann, E.T.A. (1992). Lebens-Ansichten des Katers Murr. Werke 1820-1821. En -. Sämtliche Werke in sechs Bänden. H. Steinecke; W. Segebrecht (eds.). Tomo 5. Frankfurt am Main: DKV.

"Hoffmann, E.T.A. (1985). Nachtstücke. Klein Zaches. Prinzessin Brambilla. Werke 18161820. En Sämtliche Werke in sechs Bänden. H. Steinecke; W. Segebrecht (eds.). Tomo 3. Frankfurt/M: DKV.

" Hoffmeister, G. (2004). From Goethe's Wilhelm Meister to anti-Meister Novels: The Romantic Novel between Tieck's William Lovell and Hoffmann's Kater Murr. En Mahoney, D. F. (ed.). The Literature of German Romanticism, pp. 79-99. Vol. 8 de The Camden House History of German Literature. Nueva York: Camden House.

" Jacobs, J. (1981). Wilhelm Meister und seine Brüder. Untersuchungen zum deutschen Bildungsroman. Múnich: Wilhelm Fink.

" Jauss, H. R. (1995). El proceso literario de la modernidad desde Rousseau hasta Adorno. En Las transformaciones de lo moderno. Estudios sobre las etapas de la modernidad estética, pp. 63-92. Trad. de R. Sánchez Ortiz de Urbina. Madrid: Visor.

"Koval, M. I. (2018). Vocación y renuncia: la novela de formación alemana entre la Ilustración y la Primera Guerra Mundial. Buenos Aires: Editorial de la Facultad de Filosofía y Letras, Universidad de Buenos Aires.

"Kremer, D. (2009). Lebens-Ansichten des Katers Murr (1819/21). En Kremer, D. (ed.). E.T.A. Hoffmann. Leben - Werk - Wirkung, pp. 338-356. Berlin: De Gruyter.

" Lämmert, E. (1993). Der Autor und sein Held im Roman des 19. und 20. Jahrhunderts. En The German Quarterly, vol. 66, núm. 4, pp. 415-430.

"Lukács, G. (2002). Teoría de la novela. Trad. de M. Sacristán. Madrid: Biblioteca Nacional.

"Mahoney, D. F. (1988). Der Roman der Goethezeit (1774-1829). Stuttgart: Metzler.

" Martini, F. (1961). Der Bildungsroman. En Deutsche Vierteljahrsschrift für Literaturwissenschaft und Geistesgeschichte, vol. 35, núm. 1, pp. 44-63.

"Mayer, H. (1959). Die Wirklichkeit E. Th. A. Hoffmanns. En Von Lessing bis Thomas Mann. Wandlungen der bürgerlichen Literatur in Deutschland, pp. 198-246. Württemberg: Neske.

"McGlathery, J. (1991). E.T.A. Hoffmann and the Bildungsroman. En Hardin, J. N. (ed.). Reflection and Action. Essays on the Bildungsroman, pp. 314-328. Columbia: University of South Carolina Press. 
" Meyer, H. (1961). E.T.A. Hoffmann „Lebensansichten des Katers Murr". En Das Zitat in der Erzählkunst. Zur Geschichte und Poetik des europäischen Romans, pp.114-134. Stuttgart: Metzler.

" Nehring, W. (1997). „Lebens-Ansichten des Katers Murr nebst fragmentarischer Biographie des Kapellmeisters Johannes Kreisler in zufälligen Makulaturblättern" Höhepunkt und Summe von Hoffmanns Erzählwerk. En Spätromantiker. Eichendorff und E.T.A. Hoffmann, pp. 188-214. Göttingen: Vandenhoeck und Ruprecht.

"Sammons, J. L. (1991). The Bildungsroman for Nonspecialists: An Attempt at a Clarification. En Hardin, J. N. (ed.). Reflection and Action. Essays on the Bildungsroman, pp. 26-45. Columbia: University of South Carolina Press.

"Schlegel, F. (1987). Lucinde. Trad. de B. Raposo. Valencia: Natán.

"Schön, E. (2001). Lesestoffe, Leseorte, Leserschichten. En Glaser, H. A.; Vajda, G. M. (eds.). Die Wende von der Aufklärung zur Romantik 1760-1820, pp. 77-113. Amsterdam/ Philadelphia: Benjamins.

"Segebrecht, W. (1967). Autobiographie und Dichtung. Eine Studie zum Werk E. T. A. Hoffmanns. Stuttgart: Metzler.

"Selbmann, R. (1994). Der deutsche Bildungsroman. Stuttgart: Metzler.

" Singer, H. (1963). Hoffmann. Kater Murr. En von Wiese, B. (ed.). Der deutsche Roman, tomo 1, pp. 301-328. Düsseldorf: August Bagel.

"Steinecke, H. (1992). Postfacio. En Hoffmann, E.T.A. Lebens-Ansichten des Katers Murr. Werke 1820-1821. Frankfurt/M: DKV.

"Stephan,I.(2019). Kunstepoche. En Beutin, W.etal.(eds.). DeutscheLiteraturgeschichte. Von den Anfängen bis zur Gegenwart, pp. 185-233. Stuttgart: Metzler.

"Swales, M. (1991). Irony and the Novel: Reflections on the German Bildungsroman. En Hardin, J. N. (ed.). Reflection and Action. Essays on the Bildungsroman, pp. 46-68. Columbia: University of South Carolina Press.

" Tomashevski, B. (2011). Temática. En Todorov, T. (ed.) Teoría de la literatura de los formalistas rusos, pp. 271-314. Trad. de A. M. Nethol. Buenos Aires: Siglo Veintiuno.

"Vollmer, H. (1993). Der deutschsprachige Roman 1815-1820. Múnich: Wilhelm Fink.

" Vosskamp, W. (1992). „Bildungsbücher”. Zur Entstehung und Funktion des deutschen Bildungsromans. En Schöwerling, R.; Steinecke, H. (eds.). Die Fürstliche Bibliothek Corvey: ihre Bedeutung für eine neue Sicht der Literatur des frühen 19. Jahrhunderts; Beiträge des 1. Internationalen Corvey-Symposions, 25.-27. Oktober 1990 in Paderborn, pp. 134-146. Múnich: Fink.

"Wittmann, R. (2011). ¿Hubo una revolución en la lectura a finales del siglo XVIII? En Cavallo, G.; Chartier, R. (eds.). Historia de la lectura en el mundo occidental, pp. 353387. Trad. de C. García Ohlrich. Buenos Aires: Taurus. 